\title{
Fc Gamma Receptors and Their Role in Antigen Uptake, Presentation, and T Cell Activation
}

\author{
Fabian Junker $^{1 *}$, John Gordon ${ }^{2}$ and Omar Qureshi ${ }^{2}$ \\ ${ }^{1}$ Roche Pharma Research and Early Development, Pharmaceutical Sciences, Roche Innovation Center Basel, F. \\ Hoffmann-La Roche Ltd, Basel, Switzerland, ${ }^{2}$ Celentyx Ltd, Birmingham Research Park, Birmingham, United Kingdom
}

The cellular uptake, intracellular processing, and presentation of foreign antigen are crucial processes for eliciting an effective adaptive host response to the majority of pathogens. The effective recognition of antigen by $\mathrm{T}$ cells requires that it is first processed and then presented on MHC molecules that are expressed on other cells. A critical step leading to the presentation of antigen is delivering the foreign cargo to an intracellular compartment where the antigen can be processed and loaded onto $\mathrm{MHC}$ molecules. Fc-gamma receptors (Fc $\gamma \mathrm{Rs}$ ) recognize lgG-coated targets, such as opsonized pathogens or immune complexes (ICs). Cross-linking leads to internalization of the cargo with associated activation of down-stream signaling cascades. Fc $\gamma$ Rs vary in their affinity for lgG and intracellular trafficking, and therefore have an opportunity to regulate antigen presentation by controlling the shuttling and processing of their cargos. In this way, they critically influence physiological and pathophysiological adaptive immune cell functions. In this review, we will cover the contribution of Fc $\gamma$ Rs to antigenpresentation with a focus on the intracellular trafficking of IgG-ICs and the pathways that support this function. We will also discuss genetic evidence linking Fc $\gamma R$ biology to immune cell activation and autoimmune processes as exemplified by systemic lupus erythematosus (SLE).

Keywords: Fc $\gamma$ Rs, Fc gamma receptors, antigen presentation, immune complexes, $\mathrm{T}$ cell activation, autoimmunity

\section{INTRODUCTION}

The immune response can be divided broadly into innate and adaptive immunity. Innate immune cells, such as dendritic cells, monocytes, NK cells, and neutrophils among others, mount early responses to pathogens through recognition of conserved non-host pattern antigens, such as bacterial lipopolysaccharide (LPS) or viral RNA $(1,2)$. The adaptive immune system allows a more focused response to antigen discriminating self from non-self in such a way as to limit collateral damage to host tissues yet having the capacity to recognize and evolve a response to a wider diversity of foreign antigens. $\mathrm{T}$ and B lymphocytes are key players in the adaptive immune response as a result of their ability to recognize foreign protein antigen through highly diverse specific receptors and are also capable of long lasting immune responses to the antigens they encounter $(3,4)$.

While B cells can recognize antigens in their unprocessed form, $\mathrm{T}$ cells require antigens to be processed and presented on the surface of another cell by major histocompatibility (MHC) class I or MHC class II molecules. MHC class I molecules are broadly expressed and present endogenous antigens to CD8 T cells. MHC class II molecules are expressed mostly on specialized antigenpresenting cells (APCs) and certain other cell types. These present exogenously-derived antigens to $\mathrm{CD} 4 \mathrm{~T}$ cells. Finally, there is a route whereby exogenous antigens are presented by MHC class I 
molecules termed cross-presentation (5) in which the dendritic cell (DC) plays a crucial role $(6,7)$.

DCs can broadly be grouped into three subsets in both mouse and human $(8,9)$ : Plasmacytoid DC (pDC), myeloid/conventional DC1 (cDC1), and myeloid/conventional DC2 (cDC2). The different DC subsets have varying antigen presenting capabilities, with $\mathrm{CDC1}$ cells being described as a subset with a high intrinsic capacity to cross-present antigens via $\mathrm{MHC}$ class I to activate $\mathrm{CD} 8^{+} \mathrm{T}$ cells as well as promoting $\mathrm{T}$ helper type 1 (Th1) and natural killer responses (as discussed in detail in later sections). In addition, other DC subsets, including certain cDC2 subsets (10), can also be induced to cross-present antigen. Plasmacytoid DCs, however, are largely thought to be ineffective at antigen presentation and $\mathrm{T}$ cell activation, although this issue remains somewhat controversial (11).

Inducing antigen specific $\mathrm{T}$ cell responses via MHC:antigen peptide-T cell receptor (TCR) interactions is essential for mounting long-lasting, effective immunity. This makes the uptake, subsequent intracellular processing and presentation of antigen in APCs critical. In the case of soluble protein antigens, these are to a considerable extent controlled by Fc-gamma receptor $(\mathrm{Fc} \gamma \mathrm{R})$ function, the subject of the present review.

\section{PROTEIN ANTIGENS ARE INTERNALIZED, PROTEOLYTICALLY PROCESSED, AND LOADED ONTO MHC MOLECULES INSIDE THE CELL FOR ANTIGEN PRESENTATION}

MHC molecules present antigen peptides of length 9-10 amino acids (aa) in the case of MHC class I, or 11-30aa in the case of MHC class II, held within a binding groove in the MHC molecules (12). Thus, for the bulk of extracellular antigens, proteolytic processing inside the cell is required (13).

In a healthy cell, MHC class I protein builds complexes with constituent cytoplasm-derived "self" peptides (14). Virus

\footnotetext{
Abbreviations: aa, Amino acids; ADCC, Antibody-dependent cell-mediated cytotoxicity; APC, Antigen presenting cell; BCR, B cell receptor; BLNK, Bcell linker; BM, Bone marrow; Btk, Bruton's tyrosine kinase; CD, Cluster of differentiation; cDC1, myeloid/conventional DC1; cDC2, myeloid/conventional DC2; CH, Constant region, heavy chain; CTL, Cytotoxic T lymphocyte; DAG, Diacylglycerol; DC, Dendritic cell; ELISA, Enzyme-linked immunosorbent assay; ER, Endoplasmic reticulum; Fc, Fragment, crystallizable; FcRn, Neonatal Fc receptor; $\mathrm{FcR}_{\mathrm{C}}$, Fc receptor common gamma chain; $\mathrm{Fc} \gamma \mathrm{R}, \mathrm{Fc}_{\mathrm{c}}$ gamma receptor; HEL, Henn-egg lysozyme; HKLM, Heat-killed Listeria monocytogenes; HLA, Human leukocyte antigen; IC, Immune complex; IgG, Immunoglobulin type G; IL, Interleukin; $\mathrm{IP}_{3}$, Inositol trisphosphate; ITAM, Immunoreceptor tyrosine based activation motif; ITIM, Immunoreceptor tyrosine based inhibitory motif; IVIg, Intravenous immunoglobulin; KLH, Keyhole limpet hemocyanin; KO, Knockout; lck, Lymphocyte-specific protein tyrosine kinase; LPS, Lipopolysaccharide; lyn, Lck/Yes novel tyrosine kinase; MHC, Major histocompatibility complex; NK, Natural killer cell; OVA, Ovalbumin; pDC, Plasmacytoid dendritic cell; PBMC, Peripheral blood mononuclear cell; PH, Pleckstrin homology domain; PI3K, Phosphoinositide 3-kinase; pIC, Polyinosinic:polycytidylic acid; $\mathrm{PIP}_{3}$, Phosphatidylinositol [3-5]-trisphosphate; PLC, Phospholipase C; PLD, Phospholipase D; PPD, Purified protein derivative; SH2, Src Homology 2; SLE, Systemic lupus erythematosus; SLP-76, SH2 domain containing leukocyte protein of 76kDa; Src, Sarcoma (kinase); Syk, Spleen tyrosine kinase; TAP, Transporter associated with antigen presentation; TCR, T cell receptor; Tec, Tyrosine-protein kinase; TH, T helper cell; TLR, Toll-like receptor; TNF, Tumor necrosis factor; TRIM21, Tripartite motif-containing 21; wt, Wildtype.
}

infected cells or tumor cells containing neo-antigens can however present "non-self" peptides to the T cells of the adaptive immune system resulting in their activation and culminating with the death of the unwanted host cell (15-17). Cytoplasmic proteins are initially degraded by the proteasome (18), then loaded into the endoplasmic reticulum (ER) lumen via the transporter associated with antigen processing (TAP) (19), and then incorporated into the MHC class I protein complex by the chaperone tapasin (20).

Proteolysis of antigens for MHC class II presentation occurs within the endolysosomal system and involves proteases such as cathepsins which are active at the acidic $\mathrm{pH}$ of these intracellular compartments (21). The efficiency of antigen-presentation in different cell types is related in part to the proteolytic potential of these intracellular compartments with specialized APCs containing a less acidic $\mathrm{pH}$ and protease content within the endolysosomal, favoring the conservation of peptide epitopes that can be loaded onto MHC (21). The loading of these antigenderived peptides onto MHC II requires HLA-DM to facilitate the process (22).

In the case of cross-presentation, two pathways have been described that enable MHC class I molecules to be loaded with exogenous antigen. Antigens contained in the endosomal compartment can be shuttled into the cytoplasm, where they are processed similarly to conventional cytosolic antigens relying on TAP and proteasome function (23). Alternatively, lysosomal proteases such as cathepsin $\mathrm{S}$ have been suggested to degrade exogenous antigens already in the acidic compartment (24), where peptides are then loaded to intra-endosomal MHC class I molecules. This latter cross-presentation process has been termed the "vacuolar pathway" and exists for example in certain viral or bacterial infections (25). Cross-presentation is believed to be essential for host immunity to viral infections occurring in parenchymal cells. While MHC class II molecules present peptides derived from extracellular antigens, cytoplasmic, and nuclear antigens can also gain access to MHC class II compartments (26). Entry of these antigens into the endolysosomal system for delivery to MHC II compartments can be facilitated by both Lamp-2a (27) and autophagy (28).

\section{THE ROLE OF Fc $\gamma$ Rs IN INTERNALIZING ANTIGENS}

\section{Overview of Fc $\gamma$ Rs and IgG Binding}

Fc $\gamma$ Rs bind to the IgG molecule through its Fc (fragment, crystallizable) portion (29). In humans, three groups of Fc $\gamma$ Rs have been described across a variety of cell types: Fc $\gamma$ RI, Fc $\gamma$ RIIA/B, Fc $\gamma$ RIIIA/B (30). These are expressed in differing combinations at the surface membrane of the various immune cells (31). In the case of Fc $\gamma \mathrm{RI}$, these include macrophages, neutrophils, eosinophils and DCs. For Fc $\gamma$ RIIA, cell types include macrophages, neutrophils, eosinophils, platelets, and Langerhans cells as well as conventional, but not plasmacytoid, DCs (32). Fc $\gamma$ RIIIA is found on natural killer (NK) cells and macrophages, as reviewed elsewhere (33). The inhibitory Fc gamma receptor Fc $\gamma$ RIIB is found on B cells, mast cells as well as macrophages, neutrophils, and eosinophils. Importantly, it is also expressed on cDCs (32). Flow cytometry experiments suggest that it is 
unlikely that human pDCs, in contrast to mouse pDCs where expression of the inhibitory receptor Fc $\gamma$ RIIB was claimed (34), express any $F c \gamma R s(32,35)$. Moreover, some studies on pDCs may have included contaminating $\mathrm{cDC}$ (8). Fc $\gamma$ RIIIB, which can be considered a decoy receptor since it lacks association with downstream signaling molecules (as discussed at later stages of this article), is mainly expressed on neutrophils but may under certain conditions also be expressed on other immune cells like basophils $(29,31)$.

Cell-type specific expression of mouse Fc $\gamma$ Rs (the activating Fc $\gamma$ RI, Fc $\gamma$ RIII, Fc $\gamma$ RIV, and the inhibitory Fc $\gamma$ RIIB) has also been assessed. Conventional mouse DCs were found to express Fc $\gamma$ RI, Fc $\gamma$ RIII, Fc $\gamma$ RIV, and Fc $\gamma$ RIIB $(36,37)$, while pDCs exclusively expressed Fc $\gamma$ RIIB (34). Fc $\gamma$ RIV was confirmed to be expressed on monocytes, neutrophils, and macrophages by flow cytometry (38). In addition, the inhibitory receptor Fc $\gamma$ RIIB is expressed on B cells as well as monocytes and macrophages; mouse Fc $\gamma$ RIII was described on NK cells, neutrophils, monocytes, and macrophages (31). In addition to relative expression patterns, in a recent comprehensive study using mouse and human peripheral blood, Kerntke et al. quantified the number of cell surface $F c \gamma R$ on derived immune cells including potential APCs such as B cells and monocytes (39). The authors found that on human monocytes, more Fc $\gamma$ RIIA molecules were expressed compared to Fc $\gamma$ RIIB. Likewise, in C57BL/6 mice, the activating Fc $\gamma$ RIV was the most abundant receptor on non-classical monocytes, whereas Fc $\gamma$ RIII was expressed to a much lesser degree on these cells, and Fc $\gamma$ RIIB had even less copies. Overall, these results indicate that on monocytes, under steady-state conditions, activating Fc $\gamma$ Rs are overabundantly expressed with respect to the inhibitory Fc $\gamma$ RIIB.

Importantly, mouse monocytes expressed greater numbers of the inhibitory Fc $\gamma$ RIIB compared to their human counterparts. The actual fold difference was $\sim 2.5 \mathrm{x}$ (for non-classical monocytes) and $\sim 4 \mathrm{x}$ (for classical monocytes). Such inter-species differences should be taken into account when interpreting Fc $\gamma \mathrm{R}$ subtype-specific knockout experiments as discussed in later sections of this review.

In summary, APCs in both mouse and human, express a wide array of activating (and inhibitory) Fc $\gamma$ Rs, highlighting a potential role in antigen presentation through interaction with IgG immune complexes. Consequently, antibodies of the IgG class provide the bridge between antigen and Fc $\gamma$ Rs. Fc $\gamma$ RI (also termed CD64) has the greatest affinity for IgG molecules, while Fc $\gamma$ Rs IIA/B/C (CD32) and Fc $\gamma$ RIIIA/B (CD16) have lower affinities for IgG (40). The subdivision of IgG Into 4 distinct isotypes or subclasses-IgG1, IgG2, IgG3, and IgG4-provides additional complexity to $\mathrm{Fc}$ binding interactions (41) with the precise affinity of binding dependent on the IgG isotype (40).

In a comprehensive study using selectively expressed human Fc $\gamma$ Rs, Bruhns et al. found that, as monomers, IgG1, and IgG4 exclusively bound to Fc $\gamma$ RI, whereas monomeric IgG2 failed to bind any of the Fc $\gamma$ Rs. Monomeric IgG3 also bound to Fc $\gamma$ RI and additionally to Fc $\gamma$ RIIIA. Taken together, the observations indicate that $\mathrm{Fc} \gamma \mathrm{RI}$ can be considered a high-affinity IgG receptor most likely permanently occupied by monomeric IgG in vivo (31). Bruhns et al. also studied the affinities of IgG:antigen

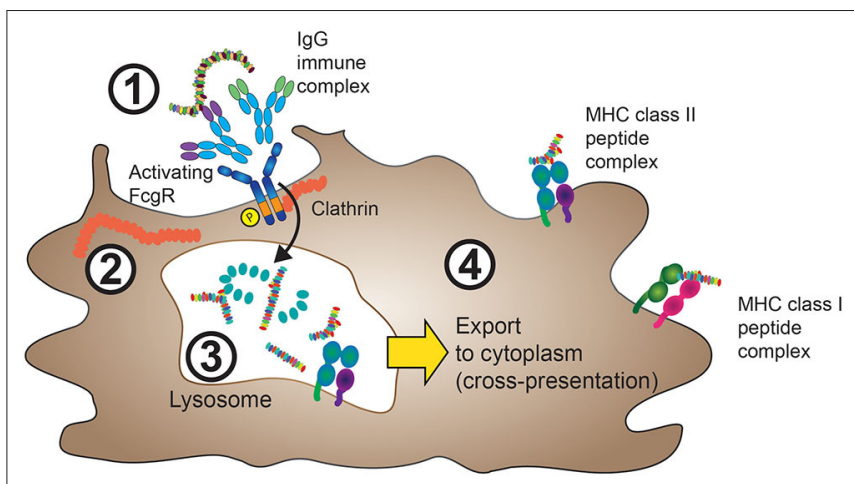

FIGURE 1 | FC $\gamma$ Rs mediate internalization of IgG-ICs. 1. IgG-ICs bind to low-affinity Fc $\gamma$ Rs at the cell surface of APCs. In the case of activating Fc $\gamma$ Rs, for instance Fc $\gamma$ RIIA, these either contain intracellular ITAM sequences (purple), or are associated with ITAM containing FcR $\gamma$ (not shown) as described in section The Molecular Machinery Downstream of Fc $\gamma R$ and Figure 3. 2. Cross-linking of activating Fc $\gamma$ Rs leads to ITAM phosphorylation and clathrin-mediated internalization of the $\mathrm{FC} \gamma \mathrm{R}$ :IgG-IC aggregate with subsequent IgG-IC transport to lysosomes. 3. Inside the lysosomal compartment, both $\mathrm{Fc} \gamma \mathrm{Rs}$ and the internalized antigen are proteolytically degraded and eventually loaded onto MHC class II complexes, processes that depend on cathepsin, and HLA-DM activity (not shown). 4. MHC class II peptide complexes are shuttled from the endolysosomal compartment to the cell membrane in order to present antigen-derived peptides (usually 13-25aa in size) to cells of the adaptive immune system. Yellow arrow: In a process termed cross-presentation, non- "self" antigens internalized through Fc $\gamma R$ cross-linking can also be shuttled to the cytoplasm, where they are processed similarly to endogenous proteins. The resulting 9-10-aa long peptides will then be incorporated in $\mathrm{MHC}$ class I protein complexes.

ICs with Fc $\gamma$ Rs. At high concentrations $(10 \mu \mathrm{g} / \mathrm{ml})$, all IgG-ICs bound to all $F c \gamma$ Rs including the inhibitory Fc $\gamma$ RIIB, although the latter was found to have a lower affinity for IgG1, IgG2, and IgG3 than other Fc $\gamma$ Rs. In summary, these findings indicate that IgG-ICs are able to differentially engage an array of Fc $\gamma$ Rs and preferentially cross-link low affinity receptors Fc $\gamma$ RIIA, IIIA, and IIB, which under serum conditions are considered the main effector Fc $\gamma$ Rs on immune cells. In addition to the different affinities of Fc $\gamma$ Rs for IgG isotypes, the IgG-IC size plays an important role in the binding to Fc $\gamma$ Rs $(35,42,43)$. Using ICs of defined sizes, the authors demonstrated that for ICs composed of IgG2 and IgG4, larger ICs exhibit increased interaction with the low-affinity human Fc $\gamma$ Rs over smaller ICs (42).

\section{Fc $\gamma R$ Directed Internalization of Exogenous Antigens}

The loading of MHC class II molecules for foreign antigen presentation, as well as the loading of MHC class I molecules for cross-presentation, occurs inside the cell. Accordingly, foreign antigens need to be internalized before they can access the processing machinery, a process that is illustrated in Figure 1.

Opsonization (from the Greek "to make tasty") of target antigens with IgG allows their detection by $F c \gamma$ receptors. The subsequent engagement of Fc $\gamma$ Rs by multivalent IgG-ICs leads to their clustering and initiation of signaling events that facilitate uptake of the antigen payload (44-47). In the case of phagocytosis 
of larger particles, actin-dependent cytoskeletal arrangements controlled by phosphatidylinositol 3-kinase, Cdc42, Rac1, and myosin motor proteins facilitate the uptake of the IgG-coated particle (48). Phagocytosis is an effective way to internalize cells as a source of antigens and allows subsequent antigenpresentation on both MHC class I and MHC class II molecules $(44,45)$. Smaller, soluble antigens bound to IgG in the form of ICs are delivered via Fc $\gamma$ Rs to lysosomes leading to degradation of both the ligand and the FcyR (49). Whilst monomeric IgG is usually shuttled between the extracellular space and the endosomal compartment via "recycling endosomes" upon internalization of the $\mathrm{Fc} \gamma \mathrm{R}(50,51)$, ICs and their associated antigen are processed intracellularly via a distinct route. Here, antigens are sorted to lysosomes for subsequent degradation and presentation (52) (Figure 1). Importantly, cross-linking of antigens is a key determinant for sorting antigen to the lysosomal compartment and preventing the recycling of an internalized IgG-IC $(49,53)$.

The uptake of soluble IgG-ICs utilizes some distinct molecular mechanisms when compared to those of phagocytosis. For example, Src kinases crucial for phagocytic uptake appear dispensable for the uptake of soluble IgG-ICs $(54,55)$. Again, in contrast to phagocytosis, the actin cytoskeleton appears not to be needed for the uptake of smaller ICs, whereas dynamin and clathrin have been found to play critical roles (56).

The Fc-mediated internalization of ICs is associated with subsequent enhancement in cellular antigen presentation efficiency (57). Expression of murine Fc $\gamma$ RIII, transfected into Fc $\gamma$ R-negative antigen-presenting B-lymphoma cells, conferred the ability to enhance the internalization of antigen-ICs in a manner dependent on tyrosine residues present within the cytoplasmic domain of the ITAM (immunoreceptor tyrosinebased activation motif)-containing FcR gamma chain (57). When these tyrosine residues were mutated, both the internalization of antigen and antigen presentation were impaired (a phenomenon that will be discussed in more detail in a later section). Of note, Fc $\gamma$ Rs appear capable of directing their cargo beyond the initial internalization step. The high affinity human IgG receptor Fc $\gamma$ RIA was found to enhance the uptake of IgG-ICs in a manner independent of its C-terminus and FcR gamma chain (58). However, whereas ICs delivered by the full-length Fc $\gamma$ RIA were directed primarily to MHC class II-containing compartments, those internalized by a truncated, tail-deleted Fc $\gamma$ RIA were diverted to recycling compartments. The tail-deleted Fc $\gamma$ RIdelivered ICs triggered inferior antigen presentation suggesting this intracellular routing of the IC may be important to the subsequent functional response (58).

Fc $\gamma$ Rs also play a critical role in antigen cross-presentation. Regnault et al. demonstrated in a mouse DC system that the internalization of IgG-ICs leads to an enhancement of crosspresentation compared to the fluid phase uptake of "naked," non-IgG opsonized antigen (59). Importantly, Fc $\gamma \mathrm{R}$ engagement of the antigen-IgG complex was critical as such enhancement in presentation was not mimicked by the DC being exposed to an irrelevant IC in conjunction with the fluid phase uptake of the antigen. This finding suggested that Fc $\gamma$ Rs had a crucial role in directing the antigen to an intracellular compartment for subsequent processing and loading onto MHC class I molecules, a concept that will be discussed in detail in the following section.

The enhancement of cross-presentation also has the potential to enhance anti-tumor immunity. Dhodapkar et al. demonstrated that when DCs internalized tumor cells coated with an antitumor IgG antibody (against syndecan 1), they were able to trigger a superior expansion of antigen specific- $\mathrm{T}$ cells compared to DCs that had internalized tumor cells alone (60). Importantly those $\mathrm{T}$ cells were also superior at killing the tumor cell targets. Blockade of Fc $\gamma$ Rs using antibodies against Fc $\gamma$ RI and Fc $\gamma$ RII inhibited the generation of antigen-specific T cells further confirming the importance of $\mathrm{Fc} \gamma \mathrm{Rs}$ in this process.

In murine DCs, the inhibitory $\mathrm{Fc} \gamma \mathrm{R}, \mathrm{Fc} \gamma \mathrm{RIIB}$, is capable of internalizing antigen-containing ICs but compared with the activating Fc $\gamma$ RIV under inflammatory conditions was found to elicit much weaker $\mathrm{T}$ cell activation, requiring a 30 -fold antigen excess to reach comparable levels (36).

In addition to the priming of $\mathrm{T}$ cells which requires the antigen to be processed for presentation on MHC molecules, mouse B cells, via the B cell receptor (BCR), recognize antigen in the unprocessed state. This process can be facilitated by Fc $\gamma$ RIIB which can recycle the antigen in an intact conformation permissive for recognition by the BCR (61). Such a mechanism has been suggested to be of particular importance in the context of humoral immunity involving $\mathrm{T}$ cell-independent $\mathrm{B}$ cell responses.

\section{FcRn Directed Shuttling of Endocytosed Antigen}

The naming of the neonatal Fc receptor $(\mathrm{FcRn})$ comes from its role in the transfer of IgG from mother to offspring across the intestinal epithelium in rats $(62,63)$ and the placenta in humans (64). However, expression of FcRn is not restricted to neonates. In fact, in adults, FcRn plays a crucial role in the maintenance of IgG levels and transport of IgG across epithelial barriers $(65,66)$ as illustrated in Figure 2. At the intestinal epithelial barrier, human FcRn can transport IgG into to the lumen to bind cognate antigen, and recycle the IgG-IC back across the intestinal barrier for subsequent uptake by mucosal DCs (67).

FcRn is predominantly detected in the endosomal compartment and constitutively cycles between there and the plasma membrane. At the low $\mathrm{pH}$ of these intracellular compartments, it binds to $\operatorname{IgG}$ with high affinity $(62,68,69)$. Internalized monomeric IgG binding to FcRn in these acidic endosomal compartments and can be recycled to the cell surface where the IgG is released upon neutral $\mathrm{pH}$ change, thereby avoiding lysosomal destruction inside the cell and extending the half-life of IgG molecules in the circulation $(70,71)$.

In human APCs such as macrophages and DCs, FcRn is co-expressed with other Fc $\gamma$ Rs (72). As FcRn is predominantly intracellular and can bind IgG at acidic $\mathrm{pH}$, it is ideally situated to engage IgG-ICs within endolysosomal compartments and to regulate IgG-IC trafficking and subsequently MHC class IImediated antigen presentation (73-78). In vitro priming of mouse $\mathrm{CD}^{+} \mathrm{T}$ cells with ICs occurs far more efficiently with DCs from wild-type mice compared to those isolated from FcRn 


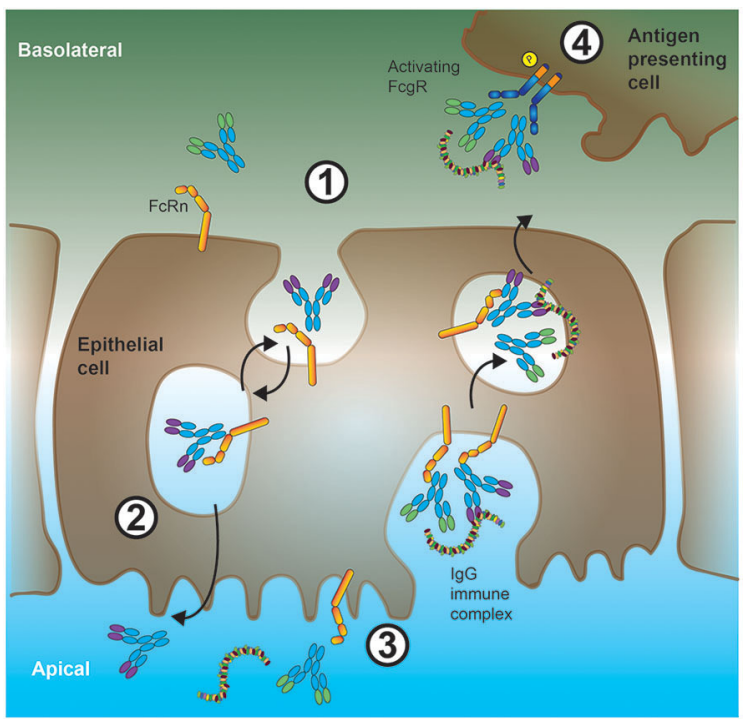

FIGURE 2 | Interplay between FcRn and low-affinity Fc $\gamma$ Rs in shuttling antigen-ICs across epithelial barriers. 1. In the intestine, IgG antibodies can bind to FcRn expressed at the basolateral cell membrane of epithelial cells. These antibodies will then be internalized and shuttled bound to FCRn with high affinity inside the acidic microenvironment of endosomes. 2. On the apical (gut lumen oriented) side of the epithelial cell, due to the more neutral $\mathrm{pH}$ and consequently, reduced affinity of FCRn for IgG, these IgG antibodies are released, and can subsequently bind to soluble protein antigen. Alternatively (orange arrow), FcRn can shuttle monomeric IgGs back to the tissue interstitium (IgG recycling), a process preventing IgG degradation in sour compartments especially in APCs. 3. Luminal IgG-ICs can be internalized and shuttled, via endosomal vesicles, by binding to FcRn, through the epithelial cell soma. 4. These IgG-ICs are then released at the basolateral cell surface to the tissue interstitium, where they bind to low-affinity Fc $\gamma$ Rs on the cell surface of APCs

knockouts (75). The importance of FcRn in antigen presentation has been further confirmed by the use of IHH-IgG. These IgG molecules contain $\mathrm{Fc}$ mutations at three critical sites, I253A, $\mathrm{H} 310 \mathrm{~A}$, and $\mathrm{H} 435 \mathrm{~A}$, which retain $\mathrm{Fc} \gamma \mathrm{R}$ binding but abrogate FcRn binding. ICs generated using IHH-IgG fail to efficiently stimulate $\mathrm{CD} 4{ }^{+}$T cells compared to those generated with wt-IgG (75). FcRn has also been demonstrated to facilitate antigen crosspresentation by the $\mathrm{CD} 8^{-} \mathrm{CD} 11 \mathrm{~b}^{+} \mathrm{DC}$ subset (73). Nevertheless, it should be noted that in some settings, the IC enhancement of a response to antigen can proceed independently of FcRn as suggested in this mouse study (79).

At epithelial barriers, FcRn can transport IgG from the basolateral side to the apical lumen. A well-characterized example is the transport of $\mathrm{IgG}$ into the intestinal epithelium (67, 80). Critically, using a human FcRn model system, once the transported IgG engages its antigen, Yoshida et al. demonstrated transport back of the IgG-containing IC across the intestinal barrier where it was taken up by DCs in the lamina propria to facilitate antigen presentation (67) as summarized in Figure 2.

This could be important from a clinical perspective where intravenous immunoglobulin (IVIg) is commonly used in the treatment of autoimmune and inflammatory diseases (81).
Through saturation of FcRn, IVIg increases clearance of pathogenic antibodies and may therefore reduce FcRn-mediated antigen-presentation as shown for example in autoimmune skin blistering diseases (82).

\section{The Molecular Machinery Downstream of Fc $\gamma R$}

The extracellular portion of Fc $\gamma$ Rs consist of two or more immunoglobulin (Ig)-like domains arranged in tandem, which interact with the $\mathrm{CH}$ (constant region, heavy chain) domains of IgG Fcs $(31,83)$. For signal transduction, Fc $\gamma$ Rs rely on tyrosine motifs in their intracellular portion, which in the case of activating Fc $\gamma$ Rs (human: Fc $\gamma$ RI, Fc $\gamma$ RIIA, Fc $\gamma$ RIIIA; mouse: Fc $\gamma$ RI, Fc $\gamma$ RIII, Fc $\gamma$ RIV) are termed immunoreceptor tyrosine-based activation motif (ITAM) (84). Fc $\gamma$ RIIA contains an intrinsic ITAM motif, whilst Fc $\gamma$ RIIIA and Fc $\gamma$ RI associate with another polypeptide, the $\mathrm{Fc}$ receptor gamma chain $(\mathrm{FcR} \gamma)$. The Fc receptor gamma chain was initially described in the context of Fce receptor signaling (85-88) and also contains an ITAM. FcR $\gamma$ is not only important for activating cell signaling downstream of Fc $\gamma$ RI and Fc $\gamma$ RIII engagement (and Fc $\gamma$ RIV in mouse), but also for the assembly and cell surface expression of the receptors (89). Fc $\gamma \mathrm{R}$ ITAM sequences are phosphorylated by scr kinases upon crosslinking leading to metabolic pathway activation and functional cell responses. Both cell type and $\mathrm{Fc} \gamma \mathrm{R}$ determine which src kinase is activated. For instance, Fc $\gamma$ RIIIA aggregation leads to lck activation in NK cells, while Fc $\gamma$ RIIA or Fc $\gamma$ RIIIA activate lyn and hck in monocytes and mast cells $(90,91)$. Subsequently, downstream of src kinase ITAM phosphorylation, src homology 2 (SH2) containing signaling molecules that bind the phosphorylated ITAM, most notably the Spleen tyrosine kinase (syk) kinase family, are recruited to the ITAM domains as illustrated in Figure 3. These events then lead to the activation of Phosphoinositide 3-kinase (PI3K) which in turn leads to production of Phosphatidylinositol [35]-trisphosphate $\left(\mathrm{PIP}_{3}\right)$ and recruitment of Pleckstrin homology (PH) domain containing molecules, such as phospholipase $\mathrm{C}$ gamma (PLC $\gamma$ ) and Tyrosine-protein kinase (Tec). Myeloid cells contain Tec kinases such as Bruton's tyrosine kinase (Btk) and Itk among others, which can be activated upon Fc $\gamma \mathrm{R}$ crosslinking. In addition, in macrophages, $\mathrm{SH} 2$ domain containing leukocyte protein of $76 \mathrm{kDa}$ (SLP-76) and B-cell linker (BLNK) link Syk activation with Btk and PLC $\gamma$ responses. Importantly, PLC $\gamma$ activity leads to the generation of second messenger molecules: cytoplasmic soluble inositol trisphosphate $\left(\mathrm{IP}_{3}\right)$ and cytoplasmic membrane bound diacylglycerol (DAG), which ultimately trigger cytoplasmic calcium mobilization and protein kinase C activation, respectively $(92,93)$. Depending on the cell type and context, Fc $\gamma$ RI, Fc $\gamma$ RIIA, and Fc $\gamma$ RIIIA aggregation can lead to degranulation, phagocytosis, antibodydependent cell-mediated cytotoxicity (ADCC), but also cytokine production and secretion of inflammatory mediators. Some of these activating $\mathrm{Fc} \gamma \mathrm{R}$ mediated effects will be discussed later in this article.

Importantly, in the case of Fc $\gamma$ RIIB, the inhibitory receptor in both mouse and human, signal transduction is not mediated 
via an ITAM domain, but via a related immunoreceptor tyrosine-based inhibition motif (ITIM) (94, 95). Here, Fc $\gamma$ RIIB crosslinking also leads to src phosphorylation of the ITIM tyrosine residues, which is followed by signaling suppressing phosphatases of the SHIP or SHP-I and SHP-II class. Importantly, since inhibitory and activating Fc $\gamma$ Rs are often co-expressed on the cell surface, it is the ratio of these molecules and the strength of the IC:Fc $\gamma \mathrm{R}$ interaction which ultimately determines if a cell is activated or repressed by ICs, a phenomenon reviewed elsewhere (96). With regards to the therapeutic exploitation of this pathway, it was demonstrated that IVIg exerts its anti-inflammatory activity through the inhibitory Fc $\gamma$ RIIB receptor (97). At least in vitro, IVIg has also been shown to inhibit MHC class II-dependent presentation of ovalbumin, albeit in this case in an $F c \gamma R$ independent manner (98).

More recently, an intracellular IgG receptor has been described, particularly in the context of antiviral immunity: namely, tripartite motif-containing 21 (TRIM21) (99). TRIM21 resides within the cytosol and recognizes the Fc-portion of IgG with high affinity. Binding of TRIM21 to antibody coated viral ICs not only results in proteosomal targeting and destruction of virions (100), but also enhanced DC maturation and crosspresentation of viral peptides to CD8 T cells (101).

In addition to mounting pro-inflammatory signaling and $\mathrm{T}$ cell activation downstream of IC challenge, human monocytederived macrophages co-incubated with high concentrations of ICs and LPS surprisingly assumed an anti-inflammatory phenotype (102). This resulted in enhanced tissue remodeling and release of angiogenetic factors, results which were confirmed in biopsies from patients with leprosy characterized by hypergammaglobulinemia and defective cell-mediated immunity. These findings suggest that macrophages may respond to IC challenge in a highly tissue and context-dependent fashion in the presence of FcgR cross-linking.

\section{Fc $\gamma R$ MEDIATED EFFECTS ON APCs: ANTIGEN UPTAKE AND T CELL PRIMING}

\section{Fc $\gamma R$ and Immune Cell Function: Evidence From Knockout Mice}

Efficient APC mediated antigen presentation to $\mathrm{T}$ cells is a crucial step in the generation of antigen specific adaptive immune responses. In the case of IC uptake, both the high-affinity Fc $\gamma$ RI, as well as the activating low-affinity Fc $\gamma$ Rs, have been shown to be of critical importance. Mechanistically, the contribution of the different Fc $\gamma$ Rs has been convincingly shown using genetic knockout (KO) mouse models.

The first definitive genetic proof that activating Fc $\gamma$ Rs contribute to IC mediated activation of DCs and subsequent MHC class II, as well as MHC class I mediated antigen presentation, was obtained by using knockout (KO) mouse models deficient for the FcR common gamma chain, FcR $\gamma$ (59). Here, the induction of DC maturation and the promotion of efficient MHC class I-restricted presentation of peptides from exogenous, IgG-complexed antigens was entirely dependent on functional FcR $\gamma$ alleles. First, an FcR $\gamma$ chain competent mouse DC cell line (D1), which expressed Fc $\gamma$ RI, Fc $\gamma$ RIIB, and Fc $\gamma$ RIII, was challenged with ovalbumin:hen egg lysozyme IC (OVA:HELICs). This effectively led to DC maturation and increase in MHC class II cell surface expression. Importantly, OVA specific CD8 ${ }^{+}$ T cell hybrid cells (B3Z) were activated by the IC challenged DCs in vitro in a TAP dependent manner, suggesting effective cross presentation subsequent to IC internalization and cell activation. Using bone marrow (BM) derived primary DCs, it was further confirmed that activating Fc $\gamma$ Rs are indeed required for the IC mediated DC activation and antigen presentation by repeating the above series of in vitro experiments using $\mathrm{FcR} \gamma \mathrm{KO}$ animals (89). Compared to wt control DCs, while the KO cells could still be activated by LPS in a similar manner, OVA-ICs failed to activate KO DCs, probably due to inefficient IC internalization.

While Regnault et al. showed that activating Fc $\gamma$ Rs critically contributed to IC uptake, other studies followed up using more candidate specific KO systems. Using a constitutive Fc $\gamma$ RIII KO mouse model, human, or sheep erythrocytes opsonized with mouse mIgG1, mIgG2a or mIgG2b were incubated with macrophages and compared to wt macrophages (103). Whereas, IgG2a and IgG2b opsonized erythrocytes were efficiently phagocytosed by both wt and mutant macrophages, IgG1 opsonized erythrocytes were much less efficiently lysed. This indicated that IgG1 preferentially bound to Fc $\gamma$ RIII expressing macrophages. Similarly, IgG1 containing, but not IgG2a or IgG2b ICs were much less efficiently trapped by follicular dendritic cells in spleens and lymph nodes of mutant animals, suggesting that this activating $\mathrm{Fc} \gamma \mathrm{Rs}$ are important in the internalization of IgG1 coated ICs.

In another mouse $\mathrm{KO}$ study, the contribution of the inhibitory Fc $\gamma$ RIIB to IC uptake was investigated. Here, Fc $\gamma$ RIIB KO BM derived DCs and macrophages were challenged with OVA-ICs and subsequent $\mathrm{T}$ cell activation was compared to wt or $\mathrm{FcR} \gamma$ deficient systems which exclusively express functional Fc $\gamma$ RIIB (104). Interestingly, Fc $\gamma$ RIIB negative DCs led to a reduced T cell activation, whereas FcR $\gamma$ deficient but Fc $\gamma$ RIIB expressing systems led to an increase in $\mathrm{T}$ cell activation. This suggested that not only activating Fc $\gamma$ Rs contributed to IC mediated T cell activation, but also the inhibitory Fc $\gamma$ RIIB, which is the only Fc $\gamma \mathrm{R}$ expressed on B cells.

In a more recent in vivo study, OVA specific mouse strains selectively deficient for Fc $\gamma$ RI/Fc $\gamma$ RII/Fc $\gamma$ RIII, Fc $\gamma$ RI/III, or Fc $\gamma$ RII only were challenged with polyclonal rabbit OVA-ICs, and subsequent CD8 OVA specific T cell responses were assessed (105). Importantly, simultaneous Fc $\gamma \mathrm{RI} / \mathrm{Fc} \gamma \mathrm{RIII} \mathrm{KO}$ did not significantly impair OVA-specific CD8 T cell proliferation after in vivo challenge thus at first glance recapitulating the findings of Yada et al. However, in mice selectively lacking the inhibitory mouse Fc $\gamma$ RIIB, there was no significant change in OVA-specific $\mathrm{T}$ cell activation compared to wt animals, suggesting that in vivo, all activating as well as the inhibitory Fc $\gamma$ RIIB could contribute to IC uptake and APC activation followed by MHC class I antigen mediated CD8 T cell activation. Consequently, several $\mathrm{Fc} \gamma \mathrm{Rs}$ may compensate for individual $\mathrm{KO}$ indicating that there is no crucial single Fc $\gamma \mathrm{R}$ critical for IC mediated T cell activation. 


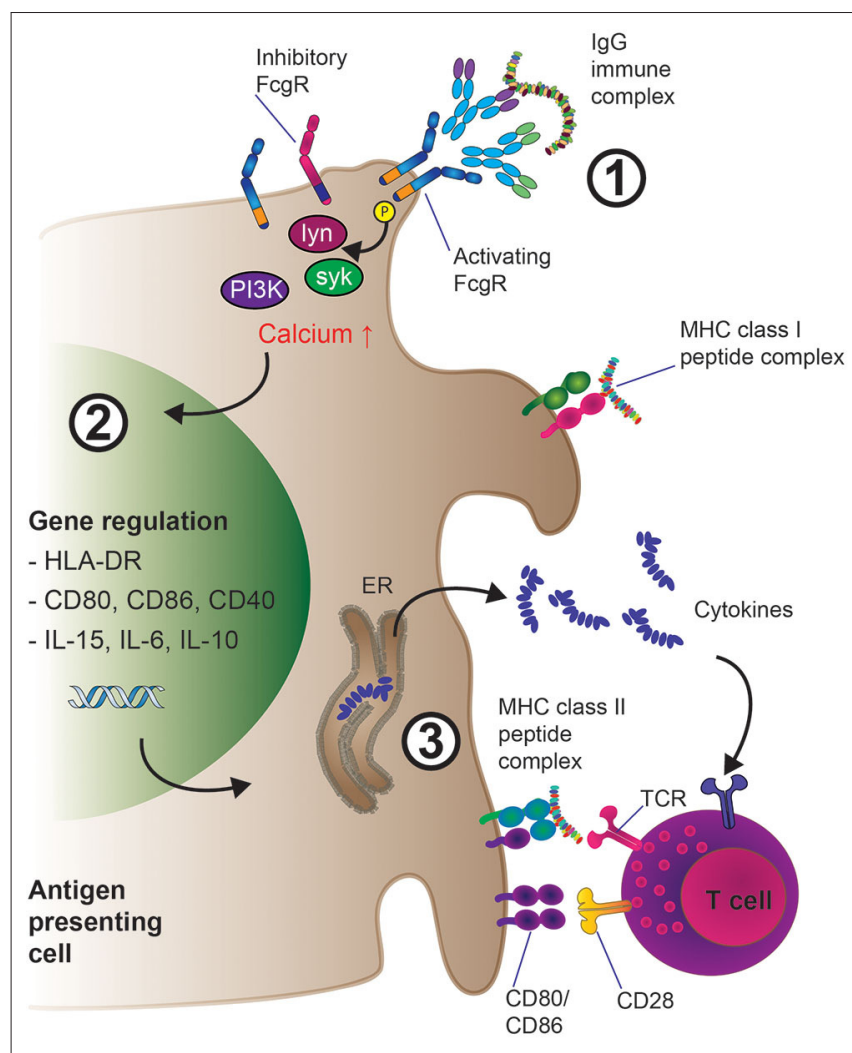

FIGURE 3 | Mechanisms of enhanced antigen presentation and T cell activation downstream of IgG-IG binding to low-affinity FC $\gamma$ Rs on APCs. 1. IgG-IC mediated cross-linking of activating low-affinity Fc $\gamma$ Rs leads to src kinase mediated ITAM phosphorylation, which induces the activation of several downstream cell-type specific kinases such as lyn, syk, and eventually PI3K, resulting in increased intracellular calcium and activation of the cell. Inhibitory (ITIM) containing Fc $\gamma$ Rs such as Fc $\gamma$ RIIB can also be expressed the cell surface; preferentially engaging inhibitory $\mathrm{Fc}_{\mathrm{c}} \mathrm{R}$ signaling would inhibit cellular activation as described in section the molecular machinery downstream of Fc $\gamma R$. 2. Cellular activation triggers transcriptional programs inside the APC such as enhanced expression of pro-inflammatory cytokines (e.g., IL-6, IL-15, $\mathrm{IL}-10)$ as well as up-regulation of membrane-bound co-activating molecules including CD80 and CD86. 3. IgG-IC mediated Fc $\gamma R$ crosslinking results in $\mathrm{MHC}$ class I and II driven presentation of antigen-derived peptides to $\mathrm{T}$ cells. Subsequent T cell activation via T cell receptor: $\mathrm{MHC}$ interaction is enhanced by concomitant co-stimulation of the $T$ cell through CD80/CD86 engagement of CD28 as well as priming of the immune response (i.e., Th1 vs. Th17) through pro-inflammatory cytokines released by the APC in the microenvironment.

The seemingly contradictory findings of Regnault et al. and Yada/de Jong et al. may be explained by the experimental conditions used in their studies. For instance, Regnault et al. focused primarily on DC biology. They employed in vitro or ex vivo assays using OVA as an antigen in the presence of ICs comprised of hen egg lysozyme (HEL) incubated with anti-HEL antibodies. These consisted of three monoclonal mouse antiHEL antibodies $(59,106)$. Yada et al., however, employed OVA (and HEL) ICs containing rabbit IgG (104). Consequently, one explanation could be that differential Fc $\gamma$ RIIB and Fc $\gamma$ RIIIA affinities for mouse vs. rabbit IgG may explain these findings. In addition, de Jong et al. performed extensive in vivo studies.
It is conceivable that the in vivo environment may be intrinsically different to the in vitro cell culture settings used by Regnault and Yada, especially considering the use of OVA-ICs consisting of non-host IgG in immunocompetent mice.

More recently, Lehmann et al. specifically investigated the role of different Fc $\gamma$ Rs on mouse splenic DC subsets (36). It was found that all subtypes of Fc $\gamma$ Rs (Fc $\gamma$ RI, Fc $\gamma$ RIIB, Fc $\gamma$ RIII, and the mouse specific Fc $\gamma$ RIV) were expressed on conventional mouse splenic DCs (cDCs). Using OVA-ICs, the authors then investigated which DC subset (and other Fc $\gamma \mathrm{R}$ expressing APCs) most efficiently internalized OVA-IC via a fluorescent quenching system allowing the distinction of internalized from total OVA-ICs. Interestingly, $\mathrm{CD} 11 \mathrm{c}^{+} \mathrm{CD}^{+}$ DCs were more efficient in the uptake of Fc $\gamma \mathrm{R}$ targeting antibodies than $\mathrm{CD} 11 \mathrm{c}^{+} \mathrm{CD} 8^{-} \mathrm{DCs}$. In contrast to monocytes, $\mathrm{B}$ cells $\left(\mathrm{Fc} \gamma \mathrm{RIIB}^{+}\right)$did not internalize any OVA-ICs in this experiment; the authors suggested that this might be due to the expression of a non-endocytic Fc $\gamma$ RIIB variant. NK and T cells did not internalize OVA-ICs, either. OVA-IC internalizing DCs then induced a short-lived T cell proliferation, which only became long-lasting if a broad adjuvant such as anti-CD40 and polyinosinic:polycytidylic acid (pIC) or heat-killed Listeria monocytogenes (HKLM) was co-applied. $\mathrm{CD} 4^{+} \mathrm{T}$ cell responses were best initiated via $\mathrm{CD} 11 \mathrm{c}^{+} \mathrm{CD} 8^{-} \mathrm{DCs}$, while $\mathrm{CD} 11 \mathrm{c}^{+} \mathrm{CD} 8^{+}$ DCs induced CD8 ${ }^{+}$T cell proliferation. T cell proliferation could be induced independently of the subtype of $\mathrm{Fc} \gamma \mathrm{R}$ via which the OVA-IC uptake occurred (including the activating Fc $\gamma$ RIV as well as the inhibitory Fc $\gamma$ RIIB).

By using a mouse with intact $F c \gamma R$ expression at the $D C$ surface but dysfunctional Fc $\gamma$ R ITAM-mediated downstream signaling [the NOTAM model (107)], T cell activation downstream of IC challenge was assessed. ITAM signaling was found to be essential in mediating effector functions such as bone marrow-derived macrophage mediated ADCC (107). Nevertheless, in the NOTAM mouse, uptake of OVA-ICs by bone marrow-derived DCs was reduced (108). Consistent with the findings of Lehmann et al. (36), MHC II antigen presentation by splenic DCs was effective in NOTAM mice, although crosspresentation was abrogated (108). Other regulators of Fc $\gamma \mathrm{R}$ signaling, such as PTPN22, a susceptibility gene for multiple autoimmune diseases, may also regulate the antigen-presentation capability of DCs from mice (109). Bone marrow DCs from PTPN22 KO mice exhibit an enhanced capability to stimulate T cells with IC-derived antigens.

Taken together, these findings suggest that both activating and inhibitory $F_{c} \gamma$ Rs have the potential to regulate the capability of APCs to activate antigen-specific T cells.

\section{Additional Mechanisms of Fc $\gamma \mathrm{R}$ Enhancement of Antigen-Presentation}

Upon Fc $\gamma \mathrm{R}$ crosslinking and antigen internalization, several important changes occur in APCs such as DCs or macrophages (110) which are illustrated in Figure 3. These include the activation and differentiation of the APC, which is accompanied by phenotypical changes, processing of the antigen (as discussed above) and the presentation of antigen-derived peptides via 
MHC class I and II molecules to T cells. T cell activation is triggered upon TCR interaction with MHC class I or MHC class II presented antigen peptide; in the course of antigen specific $\mathrm{T}$ cell activation, this first event is generally termed "signal 1." However, signal 1 on its own is not sufficient to effectively activate T cells; in addition, cell-cell interaction via cell surface receptors and ligands (co-stimulatory proteins, termed "signal 2") is required to enhance the duration of successful TCR:MHC interaction. Furthermore, cytokines present in the tissue microenvironment and secreted by the APC ("signal 3") enhance the transcriptional downstream effects of signals 1 and 2 even more and help induce specific $\mathrm{T}$ helper cell responses broadly classified by the resulting $\mathrm{T}$ cell phenotype (Th1-proinflammatory, Th2-anti-inflammatory) or by the lead cytokine secreted by the Th population, [e.g., Th17 for IL-17 secreting $\mathrm{CD}^{+}{ }^{+} \mathrm{T}$ cells $\left.(111,112)\right]$. In this respect, in recent years, it has become clear that $\mathrm{Fc} \gamma \mathrm{R}$ crosslinking through ICs dramatically enhances an APC's ability to induce all components of effective T cell stimulation: MHC-II cell surface density (signal 1); the expression of cell surface bound co-stimulatory molecules (signal 2) as well as the release of pro- or anti-inflammatory cytokines (signal 3). In this section, we will discuss crucial insights into these processes.

Firstly, $\mathrm{F} c \gamma \mathrm{R}$ crosslinking can result in increases in cell surface co-stimulatory molecules. Murine DCs incubated OVAIC upregulate not only MHC II molecules, but also the costimulatory molecule CD86 which can co-stimulate $\mathrm{T}$ cells via CD28 (59). In the same study, it was shown that CD40 is also induced at the cell surface of APCs; the CD40: CD154 axis (the CD40 ligand expressed on activated T cells) has also been shown to play an important role in T cell activation (113). These changes were monitored by flow cytometry and occurred within $24 \mathrm{~h}$ post-challenge; intriguingly, at least within this time frame, OVA alone (the "naked" antigen) was not able to induce these cell surface expression level changes, highlighting the importance of $\mathrm{Fc} \gamma \mathrm{R}$ crosslinking in the efficient activation of APCs.

In addition to providing $\mathrm{T}$ cells with co-stimulatory signals via cell surface molecules, $\mathrm{F} c \gamma \mathrm{R}$ engagement can also trigger the release of soluble factors such as cytokines that can increase or skew $\mathrm{T}$ cell activation. In a mouse study on bone marrow (BM) derived DCs challenged with mouse IgGICs or unchallenged cells, subsequent gene expression analysis by microarray furthermore corroborated the finding that ICs strongly induce $\mathrm{T}$ cell polarizing cytokine regulation (114). The authors also included a KO mouse model deficient in FcR $\gamma$. Importantly, the cytokine genes IL2, IL6, IL10, IL15, IL23a, IL27, and Ifnb1 were upregulated in WT BMDCs, but not FcR $\gamma$ chain KO BM-DCs after incubation with ICs, highlighting that the intracellular signaling cascade initiated by ITAM phosphorylation, an early event following $\mathrm{Fc} \gamma \mathrm{R}$ crosslinking, has long-lasting transcriptional changes in APC biology.

Dendritic cell phagocytosis of IgG-opsonized bacteria via Fc $\gamma$ RIIA in conjugation with TLR stimulation enhanced DC production of IL-1 $\beta$ and IL-23. These cytokines promote differentiation of Th17 cells and accordingly $\mathrm{T}$ cell responses were skewed toward a Th17 response. Other lines of evidence suggest a cross-talk between TLRs and Fc $\gamma$ Rs driving cytokine production. Exposure of human macrophages to IgG-ICs in conjunction with TLR ligands amplifies production of TNF$\alpha$, IL- $1 \beta$, and IL-6, all of which play critical roles in the pathology of diseases such as rheumatoid arthritis (RA) (115). Important differences in Fc $\gamma \mathrm{R}$-triggered cytokine production between humans and mice have been reviewed by Vogelpoel et al. (116). Finally, our own studies using human primary cellderived assay systems showed that stronger $\mathrm{T}$ cell responses to tetanus toxoid were induced by human monocyte derived DCs, or in total PBMC cultures, when challenged with the IgG-coated antigen as opposed to the "naked" antigen alone $(117,118)$. Tetanus toxoid IgG-ICs significantly enhanced the production of pro-inflammatory cytokines IFN- $\gamma$, TNF- $\alpha$, and IL- $1 \beta$, whilst Fc $\gamma$ R-blockade with a multivalent Fc-containing molecule, or with a cocktail of $F c \gamma R$ blocking antibodies, abrogated this response confirming the importance of Fc $\gamma$ Rs in mediating this enhancement.

Taken together, these findings suggest that antigen uptake, processing, and presentation, depending on the tissue microenvironment, lead to the increased expression of MHC-II and co-stimulatory cell surface molecules such as CD86 and CD40 in APCs. In addition, transcriptional up-regulation and subsequent production and release of pro-inflammatory cytokines in both mouse and human is triggered upon Fc $\gamma \mathrm{R}$ crosslinking and antigen internalization, which further enhance $\mathrm{T}$ cell polarization including pro-inflammatory Th1 and Th17 T cell responses. Furthermore, the findings in this section clearly suggests that FcrRs through IgG-ICs drive a much more pronounced and distinct type of APC activation compared to "naked" antigen uptake alone further highlighting the biological importance of these receptors.

\section{Fc $\gamma R$ Variants in Health and Disease: Evidence From Genetic Variation}

Assorted genetic variants of Fc $\gamma$ Rs have been shown to impact the affinity for and interaction with different IgG isotypes (40), a finding which has been associated with disease and clinical potential of therapeutic antibodies (119-121). In the clinic, polymorphisms often affect $\mathrm{Fc} \gamma \mathrm{R}$ function by interfering with their affinity for IgG antibodies, or their trafficking and cellular localization, which in turn has been proposed to contribute to the pathogenesis of IC driven autoimmune diseases (122) exemplified by systemic lupus erythematosus (SLE).

SLE is a chronic autoimmune disease characterized by tissue IC deposition (123) in a variety of organs including the skin and kidney (lupus nephritis), which often leads to severe clinical complications (123). In SLE, genetic predisposition has been established, and susceptibility genes linked to SLE include Fc $\gamma$ R loci including Fc $\gamma$ RII and Fc $\gamma$ RIII on chromosome 1q23 $(124,125)$. In addition, in the case of inhibitory Fc $\gamma$ RIIB, the hypothesis that receptor dysfunction may indeed be linked to SLE was initially suggested by a mouse KO model in which loss of Fc $\gamma$ RIIB resulted in spontaneous development of murine SLE (126). Since then, human studies have investigated in more detail genetic polymorphisms in SLE patients. 
In the case of the aforementioned inhibitory Fc $\gamma$ RIIB, a single amino acid polymorphism, Fc $\gamma$ RIIBT232 was initially discovered in a Japanese SLE patient population (127). This polymorphism, $695 \mathrm{~T}>\mathrm{C}$, resulted in a non-synonymous substitution, Ile232Thr (I232T), within the transmembrane domain of Fc $\gamma$ RIIB. The authors further showed that Fc $\gamma$ RIIBT232 was in some cases linked with polymorphisms affecting activating Fc $\gamma$ RIIIA. This hypothesis was experimentally tested by generating humanized mice with normal and functionally impaired Fc $\gamma$ RIIB including FcyRIIBT232 (128). Humanized mice with this mutation produced higher levels of serum IgM and IgG, and although all B cells in the peripheral blood expressed Fc $\gamma$ RIIB on their cell surface they were shown to be functionally impaired. Notably, these mice began to produce autoantibodies directed against double-stranded DNA, glucose phosphate 6-isomerase, rheumatoid factor, and made antibodies directed against cyclic citrullinated peptides, typical for SLE patients. Together, these findings suggested that in SLE, Fc $\gamma$ RIIBT232 may result in failure to inhibit activating low-affinity Fc $\gamma$ Rs, which in turn may promote a general pro-inflammatory and exaggerated humoral response to ICs.

Importantly, in a subsequent study on 1296 healthy white control subjects, only $1 \%$ of the donors were homozygous for aFc $\gamma$ RIIbT232, strengthening both its disease association as well as highlighting possible differences between ethnically different SLE patient subsets (129). In the same study, the cell biological effects of Fc $\gamma$ RIIBT232 were studied in detail (129). Using a monocytic cell line, U937, which normally only expresses activating Fc $\gamma$ RI and Fc $\gamma$ RIIA, the authors showed that activation of the cells was inhibited if they were transfected with wt Fc $\gamma$ RIIB. When challenged with Fc $\gamma$ RIIA crosslinking antibodies, U937 wt cells internalized and shuttled the cargo to cathepsin D positive lysosomes, whereas the Fc $\gamma$ RIIB transfectants showed reduced intracellular shuttling. Of note, if U937 cells were transfected with the Fc $\gamma$ RIIBT232 variant as opposed to wt Fc $\gamma$ RIIB, there was no reduction in intracellular cargo shuttling. Similarly, via antibody mediated receptor aggregation, $\mathrm{Fc} \gamma \mathrm{RI}$ mediated superoxide generation was reduced in wt, but not Fc $\gamma$ RIIBT232 transfected cells. These results were confirmed studying the phagocytosis of opsonized Streptococcus pneumonia bacteria. This strongly suggested that the FcyRIIBT232 polymorphism leads to functional impairment of Fc $\gamma$ RIIB resulting in the inability to dampen activating Fc $\gamma$ R function. Compared to its wt counterpart, Fc $\gamma$ RIIBT232 resulted in paradoxical de-phosphorylation, altered phosphatase interaction, and phosphorylation independent recruitment of SHIP. Also, Fc $\gamma$ RI triggered phospholipase D (PLD) activity, which is associated with lipid rafts (130), was inhibited by expression of Fc $\gamma$ RIIB (wt) but not Fc $\gamma$ RIIB1T232. Since both

\section{REFERENCES}

1. Leiding JW. Neutrophil evolution and their diseases in humans. Front Immunol. (2017) 8:1009. doi: 10.3389/fimmu.2017.01009

2. Medzhitov R. Recognition of microorganisms and activation of the immune response. Nature. (2007) 449:819-26. doi: 10.1038/nature06246
Fc $\gamma$ RI and Fc $\gamma$ RIIB depend on lipid rafts, membrane separation experiments were carried out showing that only wt Fc $\gamma$ RIIB, but not Fc $\gamma$ RIIBT232, was readily detectable in the lipid raft fraction of U937 cells. Conclusively, these studies show that lack of inhibitory Fc $\gamma$ RIIB function, either by complete loss of function or due to genetic polymorphisms, result in increased activating $\mathrm{Fc} \gamma \mathrm{R}$ signaling in response to ICs, thus generating an immunological environment favoring SLE.

In addition to Fc $\gamma$ RIIB, the activating Fc $\gamma$ RIIA and Fc $\gamma$ RIII have also been studied in the context of SLE (131). For Fc $\gamma$ RIIA, two co-dominantly expressed versions have been identified, R131 and H131, with the 131-Arg (R131) allele binding IgG2 much less avidity than the 131-His (H131) allele (132). Only the homozygous expression of the R/R131 variant has been associated with increased susceptibility of SLE and earlier onset (133, 134). For FcyRIII, sequencing studies suggested that a variant, Fc $\gamma$ RIIIA-176F, which displays lower binding of IgG1 and IgG3 compared to its polymorphic variant, Fc $\gamma$ RIIIA-176V, could be considered an SLE risk allele $(133,135)$.

Together, in addition to the role of polymorphisms in Fc $\gamma$ RIIB, these studies suggest that polymorphisms in activating low affinity $\mathrm{F} c \gamma \mathrm{Rs}$ may also contribute to SLE susceptibility, potentially via a shift in affinity for different IgG isotypes and which may consequently entail different immunological responses to ICs, (e.g., in lupus nephritis). However, genetic susceptibility seems to be linked to different ethnicities in SLE patient populations, and the mechanistic and cell biological effects of the genetic variants remain less well-understood.

\section{SUMMARY}

The Fc $\gamma$ R family provides a critical point of control for regulating antigen presentation of IgG-coated antigens. This control can be exerted at several stages such as the internalization of antigen, directing intracellular antigen transport, and by controlling subsequent signaling events that lead to the presentation of antigen. Stimulatory or inhibitory signals generated by Fc $\gamma$ Rs lead to regulation of co-stimulatory molecules and cytokines that further modulate the response to an antigen. The importance of these pathways to human disease is highlighted by the growing number of Fc gamma receptor genetic variants linked to human biology.

\section{AUTHOR CONTRIBUTIONS}

FJ, JG, and OQ conceptualized, wrote, and reviewed the manuscript. FJ made the article illustrations. All authors contributed to the article and approved the submitted version. 
5. Bevan MJ. Cross-priming for a secondary cytotoxic response to minor $\mathrm{H}$ antigens with $\mathrm{H}-2$ congenic cells which do not cross-react in the cytotoxic assay. J Exp Med. (1976) 143:1283-8. doi: 10.1084/jem.143. 5.1283

6. Shen Z, Reznikoff G, Dranoff G, Rock KL. Cloned dendritic cells can present exogenous antigens on both MHC class I and class II molecules. J Immunol. (1997) 158:2723-30.

7. Luckashenak N, Schroeder S, Endt K, Schmidt D, Mahnke K, Bachmann $\mathrm{MF}$, et al. Constitutive crosspresentation of tissue antigens by dendritic cells controls CD8+ T cell tolerance in vivo. Immunity. (2008) 28:52132. doi: 10.1016/j.immuni.2008.02.018

8. Balan S, Saxena M, Bhardwaj N. Dendritic cell subsets and locations. Int Rev Cell Mol Biol. (2019) 348:1-68. doi: 10.1016/bs.ircmb.2019.07.004

9. Collin M, Bigley V. Human dendritic cell subsets: an update. Immunology. (2018) 154:3-20. doi: 10.1111/imm.12888

10. Sheng J, Chen Q, Soncin I, Ng SL, Karjalainen K, Ruedl C. A discrete subset of monocyte-derived cells among typical conventional Type 2 dendritic cells can efficiently cross-present. Cell Rep. (2017) 21:120314. doi: 10.1016/j.celrep.2017.10.024

11. Embgenbroich M, Burgdorf S. Current concepts of antigen crosspresentation. Front Immunol. (2018) 9:1643. doi: 10.3389/fimmu.2018.01643

12. Wieczorek M, Abualrous ET, Sticht J, Alvaro-Benito M, Stolzenberg S, Noe F, et al. Major Histocompatibility Complex (MHC) class I and MHC class II proteins: conformational plasticity in antigen presentation. Front Immunol. (2017) 8:292. doi: 10.3389/fimmu.2017.00292

13. Delamarre L, Pack M, Chang H, Mellman I, Trombetta ES. Differential lysosomal proteolysis in antigen-presenting cells determines antigen fate. Science. (2005) 307:1630-4. doi: 10.1126/science.1108003

14. Jardetzky TS, Lane WS, Robinson RA, Madden DR, Wiley DC. Identification of self peptides bound to purified HLA-B27. Nature. (1991) 353:3269. doi: $10.1038 / 353326 \mathrm{a} 0$

15. Braunlein E, Krackhardt AM. Identification and characterization of neoantigens as well as respective immune responses in cancer patients. Front Immunol. (2017) 8:1702. doi: 10.3389/fimmu.2017.01702

16. Spies T, Cerundolo V, Colonna M, Cresswell P, Townsend A, DeMars R. Presentation of viral antigen by MHC class I molecules is dependent on a putative peptide transporter heterodimer. Nature. (1992) 355:6446. doi: $10.1038 / 355644 \mathrm{a} 0$

17. Cullis JO, Barrett AJ, Goldman JM, Lechler RI. Binding of BCR/ABL junctional peptides to major histocompatibility complex (MHC) class I molecules: studies in antigen-processing defective cell lines. Leukemia. (1994) 8:165-70.

18. Rock KL, Gramm C, Rothstein L, Clark K, Stein R, Dick L, et al. Inhibitors of the proteasome block the degradation of most cell proteins and the generation of peptides presented on MHC class I molecules. Cell. (1994) 78:761-71. doi: 10.1016/S0092-8674(94)90462-6

19. Suh WK, Cohen-Doyle MF, Fruh K, Wang K, Peterson PA, Williams DB. Interaction of MHC class I molecules with the transporter associated with antigen processing. Science. (1994) 264:1322-6. doi: 10.1126/science.81 91286

20. Rizvi SM, Raghavan M. Mechanisms of function of tapasin, a critical major histocompatibility complex class I assembly factor. Traffic. (2010) 11:33247. doi: 10.1111/j.1600-0854.2009.01025.x

21. Blum JS, Wearsch PA, Cresswell P. Pathways of antigen processing. Annu Rev Immunol. (2013) 31:443-73. doi: 10.1146/annurev-immunol-032712-095910

22. Mellins ED, Stern LJ. HLA-DM and HLA-DO, key regulators of MHCII processing and presentation. Curr Opin Immunol. (2014) 26:11522. doi: 10.1016/j.coi.2013.11.005

23. Norbury CC, Hewlett LJ, Prescott AR, Shastri N, Watts C. Class I MHC presentation of exogenous soluble antigen via macropinocytosis in bone marrow macrophages. Immunity. (1995) 3:783-91. doi: 10.1016/1074-7613(95)90067-5

24. Shen L, Sigal LJ, Boes M, Rock KL. Important role of cathepsin S in generating peptides for TAP-independent MHC class I crosspresentation in vivo. Immunity. (2004) 21:155-65. doi: 10.1016/j.immuni.2004.07.004

25. Chen L, Jondal M. Alternative processing for MHC class I presentation by immature and CpG-activated dendritic cells. Eur J Immunol. (2004) 34:952-60. doi: 10.1002/eji.200324359
26. Crotzer VL, Blum JS. Autophagy and its role in MHCmediated antigen presentation. J Immunol. (2009) 182:333541. doi: 10.4049/jimmunol.0803458

27. Zhou D, Li P, Lin Y, Lott JM, Hislop AD, Canaday DH, et al. Lamp-2a facilitates MHC class II presentation of cytoplasmic antigens. Immunity. (2005) 22:571-81. doi: 10.1016/j.immuni.2005.03.009

28. Schmid D, Munz C. Localization and MHC class II presentation of antigens targeted for macroautophagy. Methods Mol Biol. (2008) 445:21325. doi: 10.1007/978-1-59745-157-4_14

29. Ravetch JV, Bolland S. IgG Fc receptors. Annu Rev Immunol. (2001) 19:27590. doi: 10.1146/annurev.immunol.19.1.275

30. Nimmerjahn F, Ravetch JV. Fcgamma receptors as regulators of immune responses. Nat Rev Immunol. (2008) 8:34-47. doi: 10.1038/nri2206

31. Bruhns P. Properties of mouse and human IgG receptors and their contribution to disease models. Blood. (2012) 119:56409. doi: 10.1182/blood-2012-01-380121

32. Boruchov AM, Heller G, Veri MC, Bonvini E, Ravetch JV, Young JW. Activating and inhibitory IgG Fc receptors on human DCs mediate opposing functions. J Clin Invest. (2005) 115:2914-23. doi: 10.1172/JCI24772

33. Hayes JM, Wormald MR, Rudd PM, Davey GP. Fc gamma receptors: glycobiology and therapeutic prospects. J Inflamm Res. (2016) 9:20919. doi: $10.2147 /$ JIR.S121233

34. Flores M, Desai DD, Downie M, Liang B, Reilly MP, McKenzie SE, et al. Dominant expression of the inhibitory FcgammaRIIB prevents antigen presentation by murine plasmacytoid dendritic cells. J Immunol. (2009) 183:7129-39. doi: 10.4049/jimmunol.0901169

35. Patel KR, Roberts JT, Barb AW. Multiple variables at the leukocyte cell surface impact Fc gamma receptor-dependent mechanisms. Front Immunol. (2019) 10:223. doi: 10.3389/fimmu.2019.00223

36. Lehmann CHK, Baranska A, Heidkamp GF, Heger L, Neubert K, Luhr $\mathrm{JJ}$, et al. DC subset-specific induction of $\mathrm{T}$ cell responses upon antigen uptake via Fcgamma receptors in vivo. J Exp Med. (2017) 214:15091528. doi: 10.1084/jem.20160951

37. Langlet C, Tamoutounour S, Henri S, Luche H, Ardouin L, Gregoire C, et al. CD64 expression distinguishes monocytederived and conventional dendritic cells and reveals their distinct role during intramuscular immunization. J Immunol. (2012) 188:1751-60. doi: 10.4049/jimmunol.1102744

38. Nimmerjahn F, Bruhns P, Horiuchi K, Ravetch JV. FcgammaRIV: a novel FcR with distinct IgG subclass specificity. Immunity. (2005) 23:4151. doi: 10.1016/j.immuni.2005.05.010

39. Kerntke C, Nimmerjahn F, Biburger M. There is (Scientific) strength in numbers: a comprehensive quantitation of $\mathrm{Fc}$ gamma receptor numbers on human and murine peripheral blood leukocytes. Front Immunol. (2020) 11:118. doi: 10.3389/fimmu.2020.00118

40. Bruhns P, Iannascoli B, England P, Mancardi DA, Fernandez N, Jorieux $S$, et al. Specificity and affinity of human Fcgamma receptors and their polymorphic variants for human IgG subclasses. Blood. (2009) 113:371625. doi: 10.1182/blood-2008-09-179754

41. Vidarsson G, Dekkers G, Rispens T. IgG subclasses and allotypes: from structure to effector functions. Front Immunol. (2014) 5:520. doi: 10.3389/fimmu.2014.00520

42. Lux A, Yu X, Scanlan CN, Nimmerjahn F. Impact of immune complex size and glycosylation on IgG binding to human FcgammaRs. J Immunol. (2013) 190:4315-23. doi: 10.4049/jimmunol.120050

43. Robinett RA, Guan N, Lux A, Biburger M, Nimmerjahn F, Meyer AS. Dissecting FcgammaR regulation through a multivalent binding model. Cell Syst. (2018) 7:41-8.e5. doi: 10.1016/j.cels.2018.05.018

44. Kovacsovics-Bankowski M, Clark K, Benacerraf B, Rock KL. Efficient major histocompatibility complex class I presentation of exogenous antigen upon phagocytosis by macrophages. Proc Natl Acad Sci USA. (1993) 90:49426. doi: $10.1073 /$ pnas.90.11.4942

45. Mantegazza AR, Magalhaes JG, Amigorena S, Marks MS. Presentation of phagocytosed antigens by MHC class I and II. Traffic. (2013) 14:13552. doi: $10.1111 /$ tra. 12026

46. Aderem A, Underhill DM. Mechanisms of phagocytosis in macrophages. Annu Rev Immunol. (1999) 17:593 623. doi: 10.1146/annurev.immunol.17.1.593 
47. Indik ZK, Park JG, Hunter S, Schreiber AD. The molecular dissection of Fc gamma receptor mediated phagocytosis. Blood. (1995) 86:438999. doi: 10.1182/blood.V86.12.4389.bloodjournal86124389

48. Goodridge HS, Underhill DM, Touret N. Mechanisms of Fc receptor and dectin-1 activation for phagocytosis. Traffic. (2012) 13:1062-71. doi: 10.1111/j.1600-0854.2012.01382.x

49. Mellman I, Plutner H. Internalization and degradation of macrophage Fc receptors bound to polyvalent immune complexes. J Cell Biol. (1984) 98:1170-7. doi: 10.1083/jcb.98.4.1170

50. Mellman I, Plutner H, Ukkonen P. Internalization and rapid recycling of macrophage $\mathrm{Fc}$ receptors tagged with monovalent antireceptor antibody: possible role of a prelysosomal compartment. J Cell Biol. (1984) 98:11639. doi: $10.1083 /$ jcb.98.4.1163

51. Hsu VW, Bai M, Li J. Getting active: protein sorting in endocytic recycling. Nat Rev Mol Cell Biol. (2012) 13:323-8. doi: 10.1038/nrm3332

52. Ukkonen P, Lewis V, Marsh M, Helenius A, Mellman I. Transport of macrophage Fc receptors and Fc receptor-bound ligands to lysosomes. J Exp Med. (1986) 163:952-71. doi: 10.1084/jem.163.4.952

53. Mellman IS, Plutner H, Steinman RM, Unkeless JC, Cohn ZA. Internalization and degradation of macrophage $\mathrm{Fc}$ receptors during receptor-mediated phagocytosis. J Cell Biol. (1983) 96:887-95. doi: 10.1083/jcb.96.3.887

54. Fitzer-Attas CJ, Lowry M, Crowley MT, Finn AJ, Meng F, DeFranco AL, et al. Fcgamma receptor-mediated phagocytosis in macrophages lacking the Src family tyrosine kinases Hck, Fgr, and Lyn. J Exp Med. (2000) 191:66982. doi: 10.1084/jem.191.4.669

55. Davis W, Harrison PT, Hutchinson MJ, Allen JM. Two distinct regions of FC gamma RI initiate separate signalling pathways involved in endocytosis and phagocytosis. EMBO J. (1995) 14:432-41. doi: 10.1002/j.1460-2075.1995.tb07019.x

56. Tse SM, Furuya W, Gold E, Schreiber AD, Sandvig K, Inman RD, et al. Differential role of actin, clathrin, and dynamin in Fc gamma receptormediated endocytosis and phagocytosis. J Biol Chem. (2003) 278:33318. doi: 10.1074/jbc.M207966200

57. Amigorena S, Salamero J, Davoust J, Fridman WH, Bonnerot C. Tyrosinecontaining motif that transduces cell activation signals also determines internalization and antigen presentation via type III receptors for IgG. Nature. (1992) 358:337-41. doi: 10.1038/358337a0

58. van Vugt MJ, Kleijmeer MJ, Keler T, Zeelenberg I, van Dijk MA, Leusen JH, et al. The FcgammaRIa (CD64) ligand binding chain triggers major histocompatibility complex class II antigen presentation independently of its associated FcR gamma-chain. Blood. (1999) 94:80817. doi: 10.1182/blood.V94.2.808

59. Regnault A, Lankar D, Lacabanne V, Rodriguez A, Thery C, Rescigno M, et al. Fcgamma receptor-mediated induction of dendritic cell maturation and major histocompatibility complex class I-restricted antigen presentation after immune complex internalization. J Exp Med. (1999) 189:37180. doi: 10.1084/jem.189.2.371

60. Dhodapkar KM, Krasovsky J, Williamson B, Dhodapkar MV. Antitumor monoclonal antibodies enhance cross-presentation of cellular antigens and the generation of myeloma-specific killer T cells by dendritic cells. J Exp Med. (2002) 195:125-33. doi: 10.1084/jem.20011097

61. Bergtold A, Desai DD, Gavhane A, Clynes R. Cell surface recycling of internalized antigen permits dendritic cell priming of B cells. Immunity. (2005) 23:503-14. doi: 10.1016/j.immuni.2005.09.013

62. Rodewald R. pH-dependent binding of immunoglobulins to intestinal cells of the neonatal rat. J Cell Biol. (1976) 71:666-9. doi: 10.1083/jcb.71.2.666

63. Simister NE, Mostov KE. An Fc receptor structurally related to MHC class I antigens. Nature. (1989) 337:184-7. doi: 10.1038/337184a0

64. Simister NE, Story CM, Chen HL, Hunt JS. An IgG-transporting Fc receptor expressed in the syncytiotrophoblast of human placenta. Eur J Immunol. (1996) 26:1527-31. doi: 10.1002/eji.1830260718

65. Roopenian DC, Akilesh S. FcRn: the neonatal Fc receptor comes of age. Nat Rev Immunol. (2007) 7:715-25. doi: 10.1038/nri2155

66. Pyzik M, Rath T, Lencer WI, Baker K, Blumberg RS. FcRn: the architect behind the immune and nonimmune functions of $\operatorname{IgG}$ and albumin. $J$ Immunol. (2015) 194:4595-603. doi: 10.4049/jimmunol.1403014
67. Yoshida M, Claypool SM, Wagner JS, Mizoguchi E, Mizoguchi A, Roopenian DC, et al. Human neonatal Fc receptor mediates transport of IgG into luminal secretions for delivery of antigens to mucosal dendritic cells. Immunity. (2004) 20:769-83. doi: 10.1016/j.immuni.2004.05.007

68. Story CM, Mikulska JE, Simister NE. A major histocompatibility complex class I-like Fc receptor cloned from human placenta: possible role in transfer of immunoglobulin G from mother to fetus. J Exp Med. (1994) 180:237781. doi: $10.1084 /$ jem.180.6.2377

69. Kandil E, Egashira M, Miyoshi O, Niikawa N, Ishibashi T, Kasahara M. The human gene encoding the heavy chain of the major histocompatibility complex class I-like Fc receptor (FCGRT) maps to 19q13.3. Cytogenet Cell Genet. (1996) 73:97-8. doi: 10.1159/000134316

70. Simister NE, Rees AR. Isolation and characterization of an Fc receptor from neonatal rat small intestine. Eur J Immunol. (1985) 15:7338. doi: 10.1002/eji.1830150718

71. Ober RJ, Martinez C, Lai X, Zhou J, Ward ES. Exocytosis of IgG as mediated by the receptor, FcRn: an analysis at the single-molecule level. Proc Natl Acad Sci USA. (2004) 101:11076-81. doi: 10.1073/pnas.0402970101

72. Zhu X, Meng G, Dickinson BL, Li X, Mizoguchi E, Miao L, et al. MHC class I-related neonatal Fc receptor for IgG is functionally expressed in monocytes, intestinal macrophages, dendritic cells. J Immunol. (2001) 166:3266-76. doi: 10.4049/jimmunol.166.5.3266

73. Baker K, Qiao SW, Kuo TT, Aveson VG, Platzer B, Andersen JT, et al. Neonatal Fc receptor for IgG (FcRn) regulates cross-presentation of IgG immune complexes by CD8-CD11b+ dendritic cells. Proc Natl Acad Sci USA. (2011) 108:9927-32. doi: 10.1073/pnas.1019037108

74. Baker K, Rath T, Pyzik M, Blumberg RS. The role of FcRn in antigen presentation. Front Immunol. (2014) 5:408. doi: 10.3389/fimmu.2014.00408

75. Qiao SW, Kobayashi K, Johansen FE, Sollid LM, Andersen JT, Milford $\mathrm{E}$, et al. Dependence of antibody-mediated presentation of antigen on FcRn. Proc Natl Acad Sci USA. (2008) 105:9337-42. doi: 10.1073/pnas.08017 17105

76. Rath T, Baker K, Pyzik M, Blumberg RS. Regulation of immune responses by the neonatal fc receptor and its therapeutic implications. Front Immunol. (2014) 5:664. doi: 10.3389/fimmu.2014.00664

77. Stapleton NM, Einarsdottir HK, Stemerding AM, Vidarsson G. The multiple facets of FcRn in immunity. Immunol Rev. (2015) 268:25368. doi: 10.1111/imr.12331

78. Weflen AW, Baier N, Tang QJ, van den Hof M, Blumberg RS, Lencer WI, et al. Multivalent immune complexes divert FcRn to lysosomes by exclusion from recycling sorting tubules. Mol Biol Cell. (2013) 24:2398405. doi: 10.1091/mbc.e13-04-0174

79. Arnoult C, Brachet G, Cadena Castaneda D, Azzopardi N, Passot C, Desvignes $\mathrm{C}$, et al. Crucial role for immune complexes but not FcRn in immunization against anti-TNF-alpha antibodies after a single injection in mice. J Immunol. (2017) 199:418-24. doi: 10.4049/jimmunol.1601246

80. Abrahamson DR, Powers A, Rodewald R. Intestinal absorption of immune complexes by neonatal rats: a route of antigen transfer from mother to young. Science. (1979) 206:567-9. doi: 10.1126/science.493961

81. Galeotti C, Kaveri SV, Bayry J. IVIG-mediated effector functions in autoimmune and inflammatory diseases. Int Immunol. (2017) 29:49198. doi: 10.1093/intimm/dxx039

82. Li N, Zhao M, Hilario-Vargas J, Prisayanh P, Warren S, Diaz LA, et al. Complete FcRn dependence for intravenous Ig therapy in autoimmune skin blistering diseases. J Clin Invest. (2005) 115:3440-50. doi: 10.1172/JCI24394

83. Raghavan $M$, Bjorkman PJ. Fc receptors and their interactions with immunoglobulins. Annu Rev Cell Dev Biol. (1996) 12:181-220. doi: 10.1146/annurev.cellbio.12.1.181

84. Cambier JC. Antigen and Fc receptor signaling. The awesome power of the immunoreceptor tyrosine-based activation motif (ITAM). J Immunol. (1995) 155:3281-5.

85. Perez-Montfort R, Kinet JP, Metzger H. A previously unrecognized subunit of the receptor for immunoglobulin. Biochemistry. (1983) 22:57228. doi: 10.1021/bi00294a007

86. Ernst LK, Duchemin AM, Anderson CL. Association of the high-affinity receptor for IgG (Fc gamma RI) with the gamma subunit of the IgE receptor. Proc Natl Acad Sci USA. (1993) 90:6023-7. doi: 10.1073/pnas.90.13.6023 
87. Ra C, Jouvin MH, Blank U, Kinet JP. A macrophage Fc gamma receptor and the mast cell receptor for IgE share an identical subunit. Nature. (1989) 341:752-4. doi: 10.1038/341752a0

88. Brandsma AM, Hogarth PM, Nimmerjahn F, Leusen JH. Clarifying the confusion between cytokine and Fc receptor "common gamma chain". Immunity. (2016) 45:225-6. doi: 10.1016/j.immuni.2016.07.006

89. van Vugt MJ, Heijnen IA, Capel PJ, Park SY, Ra C, Saito T, et al. FcR gamma-chain is essential for both surface expression and function of human Fc gamma RI (CD64) in vivo. Blood. (1996) 87:35939. doi: 10.1182/blood.V87.9.3593.bloodjournal8793593

90. Ghazizadeh S, Bolen JB, Fleit HB. Physical and functional association of Srcrelated protein tyrosine kinases with Fc gamma RII in monocytic THP-1 cells. J Biol Chem. (1994) 269:8878-84.

91. Salcedo TW, Kurosaki T, Kanakaraj P, Ravetch JV, Perussia B. Physical and functional association of p56lck with Fc gamma RIIIA (CD16) in natural killer cells. J Exp Med. (1993) 177:1475-80. doi: 10.1084/jem.177. 5.1475

92. Mignery GA, Sudhof TC. The ligand binding site and transduction mechanism in the inositol-1,4,5-triphosphate receptor. EMBO J. (1990) 9:3893-8. doi: 10.1002/j.1460-2075.1990.tb07609.x

93. Ferris CD, Huganir RL, Supattapone S, Snyder SH. Purified inositol 1,4,5trisphosphate receptor mediates calcium flux in reconstituted lipid vesicles. Nature. (1989) 342:87-9. doi: 10.1038/342087a0

94. Vely F, Vivier E. Conservation of structural features reveals the existence of a large family of inhibitory cell surface receptors and noninhibitory/activatory counterparts. J Immunol. (1997) 159:2075-7.

95. Malbec O, Fong DC, Turner M, Tybulewicz VL, Cambier JC, Fridman WH, et al. Fc epsilon receptor I-associated lyn-dependent phosphorylation of Fc gamma receptor IIB during negative regulation of mast cell activation. $J$ Immunol. (1998) 160:1647-58.

96. Getahun A, Cambier JC. Of ITIMs, ITAMs, and ITAMis: revisiting immunoglobulin Fc receptor signaling. Immunol Rev. (2015) 268:6673. doi: 10.1111/imr.12336

97. Samuelsson A, Towers TL, Ravetch JV. Anti-inflammatory activity of IVIG mediated through the inhibitory Fc receptor. Science. (2001) 291:4846. doi: 10.1126/science.291.5503.484

98. Aubin E, Proulx DP, Trepanier P, Lemieux R, Bazin R. Prevention of T cell activation by interference of internalized intravenous immunoglobulin (IVIg) with MHC II-dependent native antigen presentation. Clin Immunol. (2011) 141:273-83. doi: 10.1016/j.clim.2011.06.009

99. Foss S, Bottermann M, Jonsson A, Sandlie I, James LC, Andersen JT. TRIM21-from intracellular immunity to therapy. Front Immunol. (2019) 10:2049. doi: 10.3389/fimmu.2019.02049

100. Mallery DL, McEwan WA, Bidgood SR, Towers GJ, Johnson CM, James LC. Antibodies mediate intracellular immunity through tripartite motifcontaining 21 (TRIM21). Proc Natl Acad Sci USA. (2010) 107:1998590. doi: 10.1073/pnas.1014074107

101. Ng PML, Kaliaperumal N, Lee CY, Chin WJ, Tan HC, Au VB, et al. Enhancing antigen cross-presentation in human monocyte-derived dendritic cells by recruiting the intracellular fc receptor TRIM21. J Immunol. (2019) 202:230719. doi: $10.4049 /$ jimmunol.1800462

102. Dalby E, Christensen SM, Wang J, Hamidzadeh K, Chandrasekaran $\mathrm{P}$, Hughitt VK, et al. Immune complex-driven generation of human macrophages with anti-inflammatory and growth-promoting activity. $J$ Immunol. (2020) 204:1901382. doi: 10.4049/jimmunol.1901382

103. Hazenbos WL, Heijnen IA, Meyer D, Hofhuis FM, Renardel de Lavalette CR, Schmidt RE, et al. Murine IgG1 complexes trigger immune effector functions predominantly via Fc gamma RIII (CD16). J Immunol. (1998) 161:3026-32.

104. Yada A, Ebihara S, Matsumura K, Endo S, Maeda T, Nakamura A, et al. Accelerated antigen presentation and elicitation of humoral response in vivo by FcgammaRIIB- and FcgammaRI/III-mediated immune complex uptake. Cell Immunol. (2003) 225:21-32. doi: 10.1016/j.cellimm.2003. 09.008

105. de Jong JMH, Schuurhuis DH, Ioan-Facsinay A, van der Voort EIH, Huizinga TWJ, Ossendorp F, et al. Murine Fc receptors for IgG are redundant in facilitating presentation of immune complex derived antigen to CD8+ $\mathrm{T}$ cells in vivo. Mol Immunol. (2006) 43:204550. doi: 10.1016/j.molimm.2006.01.002
106. Harper M, Lema F, Boulot G, Poljak RJ. Antigen specificity and crossreactivity of monoclonal anti-lysozyme antibodies. Mol Immunol. (1987) 24:97-108. doi: 10.1016/0161-5890(87)90081-2

107. de Haij S, Jansen JH, Boross P, Beurskens FJ, Bakema JE, Bos $\mathrm{DL}$, et al. In vivo cytotoxicity of type I CD20 antibodies critically depends on Fc receptor ITAM signaling. Cancer Res. (2010) 70:320917. doi: 10.1158/0008-5472.CAN-09-4109

108. Boross P, van Montfoort N, Stapels DA, van der Poel CE, Bertens C, Meeldijk J, et al. FcR $\gamma$-chain ITAM signaling is critically required for crosspresentation of soluble antibody-antigen complexes by dendritic cells. $J$ Immunol. (2014) 193:5506-14. doi: 10.4049/jimmunol.1302012

109. Clarke F, Purvis HA, Sanchez-Blanco C, Gutierrez-Martinez E, Cornish GH, Zamoyska R, et al. The protein tyrosine phosphatase PTPN22 negatively regulates presentation of immune complex derived antigens. Sci Rep. (2018) 8:12692. doi: 10.1038/s41598-018-31179-x

110. Gutcher I, Becher B. APC-derived cytokines and T cell polarization in autoimmune inflammation. J Clin Invest. (2007) 117:1119-27. doi: 10.1172/JCI31720

111. Mosmann TR, Cherwinski H, Bond MW, Giedlin MA, Coffman RL. Two types of murine helper $\mathrm{T}$ cell clone. I. Definition according to profiles of lymphokine activities and secreted proteins. 1986. J Immunol. (2005) 175:514.

112. Raphael I, Nalawade S, Eagar TN, Forsthuber TG. T cell subsets and their signature cytokines in autoimmune and inflammatory diseases. Cytokine. (2015) 74:5-17. doi: 10.1016/j.cyto.2014.09.011

113. Munroe ME, Bishop GA. A costimulatory function for T cell CD40. J Immunol. (2007) 178:671-82. doi: 10.4049/jimmunol.178.2.671

114. van Montfoort N, t Hoen PA, Mangsbo SM, Camps MG, Boross P, Melief CJ, et al. Fcgamma receptor IIb strongly regulates Fcgamma receptorfacilitated T cell activation by dendritic cells. J Immunol. (2012) 189:92101. doi: 10.4049/jimmunol.1103703

115. Vogelpoel LT, Hansen IS, Rispens T, Muller FJ, van Capel TM, Turina $\mathrm{MC}$, et al. Fc gamma receptor-TLR cross-talk elicits pro-inflammatory cytokine production by human M2 macrophages. Nat Commun. (2014) 5:5444. doi: $10.1038 /$ ncomms6444

116. Vogelpoel LT, Baeten DL, de Jong EC J, den Dunnen. Control of cytokine production by human fc gamma receptors: implications for pathogen defense and autoimmunity. Front Immunol. (2015) 6:79. doi: 10.3389/fimmu.2015.00079

117. Junker F, Krishnarajah S, Qureshi O, Humphreys D, Fallah-Arani F. A simple method for measuring immune complex-mediated, Fc gamma receptor dependent antigen-specific activation of primary human T cells. J Immuno Methods. (2018) 454:32-39. doi: 10.1016/j.jim.2017.12.002

118. Qureshi OS, Rowley TF, Junker F, Peters SJ, Crilly S, Compson J, et al. Multivalent Fcgamma-receptor engagement by a hexameric Fc-fusion protein triggers Fcgamma-receptor internalisation and modulation of Fcgamma-receptor functions. Sci Rep. (2017) 7:17049. doi: 10.1038/s41598-017-17255-8

119. Chan KR, Ong EZ, Mok DZ, Ooi EE. Fc receptors and their influence on efficacy of therapeutic antibodies for treatment of viral diseases. Expert Rev Anti Infect Ther. (2015) 13:1351-60. doi: 10.1586/14787210.2015.1079127

120. Siberil S, Dutertre CA, Fridman WH, Teillaud JL. FcgammaR: the key to optimize therapeutic antibodies? Crit Rev Oncol Hematol. (2007) 62:2633. doi: 10.1016/j.critrevonc.2006.12.003

121. Mellor JD, Brown MP, Irving HR, Zalcberg JR, Dobrovic A. A critical review of the role of $\mathrm{Fc}$ gamma receptor polymorphisms in the response to monoclonal antibodies in cancer. J Hematol Oncol. (2013) 6:1. doi: 10.1186/1756-8722-6-1

122. Nagelkerke SQ, Schmidt DE, de Haas M, Kuijpers TW. Genetic variation in low-to-medium-affinity Fcgamma receptors: functional consequences, disease associations, and opportunities for personalized medicine. Front Immunol. (2019) 10:2237. doi: 10.3389/fimmu.2019.02237

123. Toong C, Adelstein S, Phan TG. Clearing the complexity: immune complexes and their treatment in lupus nephritis. Int J Nephrol Renovasc Dis. (2011) 4:17-28. doi: 10.2147/IJNRD.S10233

124. Wakeland EK, Liu K, Graham RR, Behrens TW. Delineating the genetic basis of systemic lupus erythematosus. Immunity. (2001) 15:397408. doi: 10.1016/S1074-7613(01)00201-1 
125. Pradhan V, Deshpande N, Nadkarni A, Patwardhan M, Surve P, Ghosh K. Fc gamma R IIIB polymorphisms: their association with clinical manifestations and autoantibodies in SLE patients from western India. Int J Rheum Dis. (2010) 13:138-43. doi: 10.1111/j.1756-185X.2010.01469.x

126. Bolland S, Ravetch JV. Spontaneous autoimmune disease in Fc(gamma)RIIB-deficient mice results from strain-specific epistasis. Immunity. (2000) 13:277-85. doi: 10.1016/S1074-7613(00)00027-3

127. Kyogoku C, Dijstelbloem HM, Tsuchiya N, Hatta Y, Kato H, Yamaguchi A, et al. Fcgamma receptor gene polymorphisms in Japanese patients with systemic lupus erythematosus: contribution of FCGR2B to genetic susceptibility. Arthritis Rheum. (2002) 46:1242-54. doi: 10.1002/art.10257

128. Baerenwaldt A, Lux A, Danzer H, Spriewald BM, Ullrich E, Heidkamp G, et al. Fcgamma receptor IIB (FcgammaRIIB) maintains humoral tolerance in the human immune system in vivo. Proc Natl Acad Sci USA. (2011) 108:18772-7. doi: 10.1073/pnas.1111810108

129. Floto RA, Clatworthy MR, Heilbronn KR, Rosner DR, MacAry PA, Rankin A, et al. Loss of function of a lupus-associated FcgammaRIIb polymorphism through exclusion from lipid rafts. Nat Med. (2005) 11:10568. doi: $10.1038 / \mathrm{nm} 1288$

130. Diaz O, Mebarek-Azzam S, Benzaria A, Dubois M, Lagarde M, Nemoz G, et al. Disruption of lipid rafts stimulates phospholipase d activity in human lymphocytes: implication in the regulation of immune function. J Immunol. (2005) 175:8077-86. doi: 10.4049/jimmunol.175.12.8077

131. Pradhan V, Patwardhan M, Ghosh K. Fc gamma receptor polymorphisms in systemic lupus erythematosus and their correlation with the clinical severity of the disease. Indian J Hum Genet. (2008) 14:77-81. doi: 10.4103/0971-6866.44998

132. Jiang XM, Arepally G, Poncz M, McKenzie SE. Rapid detection of the Fc gamma RIIA-H/R 131 ligand-binding polymorphism using an allele-specific restriction enzyme digestion (ASRED). J Immunol Methods. (1996) 199:559. doi: 10.1016/S0022-17599600164-0

133. Magnusson V, Johanneson B, Lima G, Odeberg J, Alarcon-Segovia $\mathrm{D}$, Alarcon-Riquelme $\mathrm{ME}$, et al. Both risk alleles for FcgammaRIIA and FcgammaRIIIA are susceptibility factors for SLE: a unifying hypothesis. Genes Immun. (2004) 5:130-7. doi: 10.1038/sj.gene.63 64052

134. Bazilio AP, Viana VS, Toledo R, Woronik V, Bonfa E, Monteiro RC. FC gamma RIIa polymorphism: a susceptibility factor for immune complexmediated lupus nephritis in Brazilian patients. Nephrol Dial Transplant. (2004) 19:1427-31. doi: 10.1093/ndt/gfh121

135. Karassa FB, Trikalinos TA, Ioannidis JP, Fc gamma RIIIA-SLE meta-analysis investigators. The Fc gamma RIIIA-F158 allele is a risk factor for the development of lupus nephritis: a meta-analysis. Kidney Int. (2003) 63:147582. doi: 10.1046/j.1523-1755.2003.00873.x

Conflict of Interest: This work was conceived out of personal dedication as a joint effort between FJ, OQ, and JG and was not funded by their employers. FJ is currently an employee of F. Hoffmann-La Roche Ltd, and a former employee of UCB Pharma. OQ is an employee of Celentyx Ltd and a former employee of UCB Pharma. JG is currently an owner and Director of Celentyx Ltd.

Copyright (C) 2020 Junker, Gordon and Qureshi. This is an open-access article distributed under the terms of the Creative Commons Attribution License (CC BY). The use, distribution or reproduction in other forums is permitted, provided the original author(s) and the copyright owner(s) are credited and that the original publication in this journal is cited, in accordance with accepted academic practice. No use, distribution or reproduction is permitted which does not comply with these terms. 